Artículo original

\title{
Distribución y caracterización fenotípica y genotípica de Listeria monocytogenes en aislamientos de alimentos, Colombia, 2010-2018
}

Ana Isabel Muñoz, Edna Catering Rodríguez

Instituto Nacional de Vigilancia de Medicamentos y Alimentos, INVIMA, Bogotá, D.C., Colombia

Introducción. Listeria monocytogenes es un patógeno transmitido por alimentos que causa infecciones en humanos, entre ellas, meningitis, meningoencefalitis y septicemias, así como abortos. Con la tipificación serológica se han identificado 13 serotipos, siendo el $4 \mathrm{~b}$ el causante de la mayoría de los brotes en el mundo.

Objetivo. Determinar la frecuencia y la distribución de los serotipos y subtipos moleculares de L. monocytogenes aislados de alimentos en Colombia entre el 2010 y el 2018.

Materiales y métodos. Se hizo un estudio descriptivo y retrospectivo a partir del análisis de 2.420 aislamientos que fueron identificados como L. monocytogenes y otras especies, por medio de pruebas bioquímicas, serológicas y de subtipificación molecular mediante electroforesis en gel de campo pulsado (PFGE).

Resultados. De los 2.420 aislamientos recibidos, 2.326 fueron confirmados como $L$. monocytogenes. Los serotipos encontrados fueron: 4b (52\%), 4d-4e (14,5 \%), 1/2a (11 \%), $1 / 2 c(9,4 \%), 1 / 2 b(9 \%)$, y 3a, 3b, 3c, 4c, 4d, 4e y 7 (menos de $2 \%)$. Procedían de Bogotá (43\%), Antioquia (25\%), Valle (10\%), Nariño (9\%) y otros departamentos $(7 \%)$. La caracterización genotípica agrupó los aislamientos evaluados en 167 patrones de PFGE; los perfiles más frecuentes se presentaron en productos lácteos, cárnicos y alimentos preparados.

Recibido: 24/05/2021

Aceptado: $02 / 08 / 2021$

Publicado: 03/08/2021

Citación:

Muñoz Al, Rodríguez EC. Distribución y caracterización fenotípica y genotípica de Listeria monocytogenes en aislamientos de alimentos, Colombia, 2010-2018. Biomédica. 2021;41(Supl.2):165-79.

https://doi.org/10.7705/biomedica.6152

\section{Correspondencia:}

Ana Isabel Muñoz, Instituto Nacional de Vigilancia de Medicamentos y Alimentos-INVIMA, Carrera $10 \mathrm{~N}^{\circ}$

64-28, Bogotá, D.C., Colombia

Teléfono: (310) 2702147

anisabel2001@yahoo.com

Contribución de los autores:

Ana Isabel Muñoz realizó el diseño del estudio y la elaboración del manuscrito, la producción de antisueros factoriales de Listeria spp., la caracterización fenotípica, la emisión de resultados y el análisis de ellos; el diseño del tamaño de la muestra y el muestreo aleatorio de los aislamientos de Listeria monocytogenes para análisis de PFGE.

Edna Catering Rodríguez estandarizó la metodología de PFGE en Listeria monocytogenes y participó en la elaboración del manuscrito.

\section{Financiación:}

Este estudio fue financiado por el Instituto Nacional de Vigilancia de Medicamentos y Alimentos como parte de sus actividades misionales.

\section{Conflicto de intereses:}

Las autoras declaramos que no hubo ningún conflicto de intereses durante la realización del trabajo.
Conclusión. El 96,1 \% de los aislamientos correspondieron a L. monocytogenes, con una buena concordancia entre el aislamiento y la identificación; el serotipo 4b, extremadamente virulento, fue el más frecuente. El análisis molecular evidenció la posible diseminación y permanencia en el tiempo de varios serotipos, lo que resalta la importancia de incluir este patógeno en los programas de vigilancia epidemiológica en alimentos.

Palabras clave: Listeria monocytogenes; enfermedades transmitidas por alimentos; electroforesis en gel de campo pulsado.

\section{Distribution and phenotypic and genotypic characterization of Listeria monocytogenes isolated from food, Colombia, 2010-2018}

Introduction: Listeria monocytogenes is a food-borne pathogen that may cause infections in humans such as meningitis, meningoencephalitis, and septicemia, as well as abortions. By serological typing 13 serotypes have been identified of which $4 \mathrm{~b}$ is responsible for most of the outbreaks in the world.

Objective: To determine the frequency and distribution of serotypes and molecular subtypes of L. monocytogenes isolated in Colombia from food from 2010 to 2018. Materials and methods: We conducted a retrospective and descriptive study based on the analysis of 2,420 isolates confirmed as L. monocytogenes and other species using biochemical and serological tests, and pulsed-field gel electrophoresis (PFGE) for molecular subtyping. Results: Of the 2,420 isolates received, 2,326 were confirmed as L. monocytogenes. The serotypes found were $4 \mathrm{~b}(52 \%), 4 d-4 \mathrm{e}(14.5 \%), 1 / 2 \mathrm{a}(11 \%), 1 / 2 \mathrm{c}(9.4 \%), 1 / 2 \mathrm{~b}(9 \%)$, and $3 a, 3 b, 3 c, 4 c, 4 d, 4 e$ and 7 (less than $2 \%)$. The isolates came from Bogotá (43\%), Antioquia (25\%), Valle (10\%), Nariño $(9 \%)$, and other departments $(7 \%)$. The genotypic characterization grouped the isolates in 167 PFGE patterns. The most frequent patterns were identified in various dairy and meat products, and in prepared foods.

Conclusion: A $96.1 \%$ of the isolates corresponded to L. monocytogenes showing good agreement between isolates and identification. Serotype $4 \mathrm{~b}$, highly virulent, was the most frequent. The molecular analysis showed the possible dissemination and permanence over time of several serotypes, which highlights the importance of including this pathogen in epidemiological food surveillance programs.

Keywords: Listeria monocytogenes; foodborne diseases; electrophoresis, gel, pulsed-field. 
El género Listeria está conformado por bacterias Gram positivas con un bajo contenido de $G+C$ (1). Hasta 1984, se incluían en él seis especies (1): $L$. monocytogenes, L. innocua, L. seeligeri, L. welshimeri, L. grayi y L. ivanovii (26). En el 2009, se describieron otras dos especies: L. rocourtiae y L. marthii (7$8)$, y posteriormente, se reconocieron nueve más: $L$. aquatica, $L$. cornellensis, L. floridensis, L. grandensis, L. riparia, L. booriae, L. newyorkensis, $L$. fleischmannii y L. weihenstephanensis (9-12). Recientemente, mediante estudios filogenéticos y a partir de la información de la secuencia genómica completa, se identificaron las siguientes nuevas especies: $L$. costaricencis, $L$. goaensis, $L$. thailandesis, $L$. valentina, $L$. cossartiae, L. farberi, $L$. immoviles, $L$. portnoyi y L. rustica (13-17).

Listeria monocytogenes se considera una especie patógena para los humanos y los animales, y L. ivanovii, para los animales (1). No obstante, existen reportes de listeriosis humanas ocasionadas por L. ivanovi y L. innocua $(1,18,19)$.-L. monocytogenes es un microorganismo ubicuo, facultativo, intracelular y oportunista, que causa graves infecciones en humanos y animales (1). Su principal ruta de transmisión es el consumo de alimentos contaminados (20). Tiene la capacidad de adaptarse a condiciones extremas, lo que permite su supervivencia en la cadena de producción de una gran variedad de alimentos de origen animal y vegetal $(20,21)$. Puede ocasionar grandes pérdidas económicas en la industria a nivel nacional e internacional, dada la obligación de retirar del mercado los alimentos contaminados con el microorganismo $(22,23)$.

Este patógeno causa infecciones sistémicas graves, como meningitis, meningoencefalitis, encefalitis y septicemia $(1,20,24-26)$. La diseminación bacteriana subclínica puede causar endocarditis, e infecciones cutáneas, osteoarticulares o de las vías biliares (24). Además, las infecciones de la madre y del recién nacido pueden producir aborto espontáneo, meningitis, septicemia neonatal e, incluso, la muerte fetal $(1,20,24-27)$.

Listeria monocytogenes ocupa el tercer lugar como agente etiológico causante de meningitis neonatal, con una letalidad de hasta el $60 \%(27)$. Las mujeres embarazadas tienen diez veces más probabilidades de contraer infecciones por listeriosis que otras personas (25).

A pesar de que la listeriosis continúa siendo una enfermedad poco frecuente, con una morbilidad baja comparada con otras enfermedades transmitidas por alimentos, la mortalidad por esta causa está entre el $20 \mathrm{y} \mathrm{el}$ $30 \%(1,23,26,28)$, y en el $95 \%$ de los casos requiere hospitalización (28).

En el mundo se han reportado muchos brotes de listeriosis humana asociados con diferentes tipos de alimentos, como quesos y otros derivados lácteos, frutas (melones), productos cárnicos, mariscos y pescados ahumados $(20,29-36)$.

Para identificar las cepas de L. monocytogenes, se utilizan diferentes métodos fenotípicos como las pruebas bioquímicas de identificación y confirmación de especies (37); y la de tipificación serológica, muy útil para determinar los serotipos mediante el esquema de Paterson. Esta metodología, que fue modificada por Seeliger y Höhne $(38,39)$ y aún está vigente, ha permitido caracterizar 13 serotipos de la especie: 1/2a, 1/2b, 1/2c, 3a, 3b, 3c, 4a, 4ab, 4b, 4c, 4d, 4e y $7(1,23,26,28,38,39)$. 
Para la caracterización genotípica de L. monocytogenes en este estudio, se utilizó la electroforesis en gel de campo pulsado (Pulsed-Field Gel Electrophoresis, PFGE). En el estudio multicéntrico de subtipificación de $L$. monocytogenes de la Organización Mundial de la Salud (OMS) de 1996, se determinó que la PFGE era un método preciso y reproducible para comparar molecularmente los aislamientos de este agente patógeno, utilizando las enzimas de restricción Ascl y Apal (40).

En Colombia, la listeriosis no es una enfermedad de notificación obligatoria; sin embargo, en el periodo 2010-2018 se notificaron al Sistema de Vigilancia en Salud Pública (Sivigila) 42 brotes de enfermedad transmitida por alimentos ocasionados por L. monocytogenes, los cuales afectaron a 1.255 personas por el consumo de alimentos como quesos frescos, derivados cárnicos, comidas de restaurantes y, en general, alimentos listos para el consumo (41).

Para el estudio, se hizo una búsqueda institucional activa en el Sistema de Información de Apoyo a la Notificación e Investigación de Eventos de Interés en Salud Pública (SIANIESP) del Sivigila en los registros individuales de prestación de servicios de salud (RIPS), para: listeriosis cutánea (A230), otras formas de listeriosis (A328), listeriosis no específica (A329), listeriosis congénita (P372), meningitis y meningoencefalitis (A321), y septicemias (A327). Hubo un total de 696 consultas externas por listeriosis, de las cuales 487 fueron por meningitis y meningoencefalitis, y 209 por las otras formas de listeriosis. Asimismo, en este periodo los servicios de urgencias notificaron al Sivigila 85 casos de listeriosis (42).

En Colombia, la meningitis bacteriana es una enfermedad de notificación obligatoria cuando el agente causal es Haemophillus influenzae, Streptococcus pneumonie o Neisseria meningitidis, pero no cuando es L. monocytogenes, lo cual ocasiona un vacío en la vigilancia de este microorganismo (43). El Instituto Nacional de Vigilancia de Medicamentos y Alimentos (Invima), consciente de la gravedad de la listeriosis y teniendo en cuenta que aún no está contemplada en la vigilancia de los alimentos, ha incluido L. monocytogenes como un indicador obligatorio de inocuidad en la vigilancia de los alimentos que se producen o se consumen en el país. Esta vigilancia es rutinaria y es ejercida por las entidades territoriales; además, el envío de los aislamientos de los laboratorios de salud pública al laboratorio de referencia del Invima para su confirmación, serotipificación y subtipificación molecular, es de obligatorio cumplimiento.

En este contexto, los objetivos de este estudio fueron confirmar el resultado de los aislamientos de $L$. monocytogenes enviados por los laboratorios de salud pública, y determinar la frecuencia y distribución de los serotipos y subtipos moleculares que circulan en el país procedentes de los alimentos que son motivo de vigilancia, como también, los de aquellos asociados epidemiológicamente con los brotes en el periodo comprendido entre el 2010 y el 2018.

\section{Materiales y métodos}

\section{Tipo de estudio}

Se hizo un estudio descriptivo y retrospectivo entre el 2010 y el 2018 para confirmar la presencia de L. monocytogenes en los aislamientos recibidos en el Laboratorio de Microbiología de Alimentos del Invima. 


\section{Muestra}

Durante este periodo, se recibieron 2.420 aislamientos de $L$. monocytogenes enviados por los laboratorios de alimentos de los departamentos adscritos a los de salud pública (LDSP) de Arauca, Antioquia, Atlántico, Bolívar, Boyacá, Caldas, Caquetá, Casanare, Cauca, Cesar, Córdoba, Cundinamarca, Bogotá, La Guajira, Magdalena, Meta, Nariño, Norte de Santander, Putumayo, Quindío, Risaralda, San Andrés Islas, Santander, Sucre y Valle del Cauca, además de los aislamientos recuperados en el laboratorio de referencia. Los servicios de Amazonas, Guainía, Guaviare, Huila, Tolima, Vaupés y Vichada, no enviaron ningún aislamiento.

En el análisis de las variables, se utilizó el programa Microsoft Office Excel $2013^{\circledR}$ para calcular las frecuencias y las proporciones, y luego se hicieron un análisis univariado y uno bivariado de los datos del estudio. Se incluyeron las siguientes variables: categoría de alimentos, alimentos, procedencia, serotipos y patrones de PFGE.

\section{Caracterización fenotípica}

Para identificar los aislamientos se siguió el procedimiento del manual de bacteriología analítica (Bacteriologycal Analytical Manual): coloración de Gram, determinación de catalasa, pruebas bioquímicas (ramnosa, xilosa y manitol), crecimiento en forma de sombrilla en agar, motilidad, pruebas de hemólisis y prueba de Christie-Atkins-Much-Peterson (CAMP). Asimismo, se utilizó el sistema bioquímico API Listeria de Biomérieux (37).

Para la tipificación serológica de Listeria spp., se siguió el protocolo de Seeliger y Höhne (38) desarrollado por el Laboratorio de Listeria del Instituto Pasteur en París, Francia (44). Se incluyeron antisueros de factores somáticos y flagelares producidos por el Laboratorio de Microbiología de Alimentos del Invima, y se utilizaron cepas de referencia del Instituto Pasteur de París mediante la inmunización de conejos Nueva Zelanda; asimismo, se utilizaron los antisueros comerciales Eurobio ${ }^{\mathrm{TM}}$ y Denka Seiken ${ }^{\mathrm{TM}}$.

El control externo de calidad de la identificación y serotipificación, y su seguimiento, estuvieron a cargo del Laboratorio de Listeria y del Centro Colaborativo de la OMS para la listeriosis de origen alimentario del Instituto Pasteur de París.

Los alimentos de los cuales se aisló L. monocytogenes se clasificaron según su categoría y procedencia.

\section{Caracterización genotípica}

Los aislamientos de L. monocytogenes se caracterizaron con la técnica de PFGE ya descrita (45). Se seleccionó la enzima de restricción Apal, teniendo en cuenta que el poder discriminatorio calculado mediante el índice de Simpson no difiere entre las dos enzimas recomendadas, Apal y Ascl, cuando se evalúa L. monocytogenes (46); además, se ha demostrado que el número de perfiles únicos observado con Apal es mayor que el obtenido con Ascl (47), y se ha evidenciado que el análisis del serotipo 4b con la enzima Apal tiene mayor poder de discriminación que otros métodos de tipificación, incluida la restricción con Ascl (48). Los dendrogramas se generaron con el programa Bionumérics ${ }^{\mathrm{TM}}$, versión 7.5 (Applied Maths, Bélgica) mediante el método UPGMA con una optimización de tolerancia de 1,5\%. 
La muestra para seleccionar los aislamientos sometidos a PFGE, se calculó utilizando Epi-Info, versión 7.2, de 2018, con una estimación del 50 \% (por ser desconocida), un error esperado del $5 \%$ y una significación del $95 \%$. Se le atribuyó un peso porcentual a cada categoría de alimento y a la frecuencia de sus serotipos. La selección fue aleatoria y quedó conformada por 368 aislamientos distribuidos así: leche y derivados lácteos $(n=188)$, carne y derivados cárnicos $(n=93)$, alimentos listos para el consumo $(n=30)$, aves $(n=15)$, muestras de plantas procesadoras de alimentos $(n=3)$, y productos de panadería y pastelería $(n=1)$. Además, se analizaron todos los aislamientos $(n=38)$ implicados en casos y brotes de enfermedades transmitidas por alimentos.

\section{Resultados}

De los 2.420 aislamientos recibidos, L. monocytogenes se confirmó en $2.326(96,1 \%)$ y se identificaron otras especies del género Listeria en 94 (3,9\%). De los 2.326 de L. monocytogenes, 2.288 (98,4 \%) provenían de alimentos que son objeto de los programas de vigilancia del Invima como parte del control de calidad de alimentos en el país, y los $38(1,6 \%)$ restantes correspondían a casos y brotes de enfermedades transmitidas por alimentos.

Los alimentos remitidos y confirmados como L. monocytogenes por el laboratorio del Invima procedían, con mayor frecuencia, de Bogotá, Antioquia y Valle del Cauca (figura 1).

Las categorías de alimentos más frecuentemente contaminados por $L$. monocytogenes fueron las leches y los derivados lácteos ( $n=1.317 ; 56,6 \%)$, las carnes y los derivados cárnicos $(n=659 ; 28,3 \%)$ y los alimentos listos para el consumo ( $n=209 ; 9 \%$ ) (cuadro suplementario 1 ).

Las categorías de alimentos en los que más frecuentemente se aisló $L$. monocytogenes fueron las leches y los derivados lácteos, con 19,91\% en Antioquia y $17,41 \%$ en Bogotá, y las carnes y derivados cárnicos, con 17,37 \% en Bogotá (cuadro suplementario 2).



Figura 1. Presencia de Listeria monocytogenes aislada de alimentos según su procedencia, Colombia 2010-2018 
Durante el periodo de estudio, se determinaron 12 serotipos. El serotipo más frecuente fue el $4 b(n=1.208)$ (figura 2$)$, y la mayor incidencia de este serotipo se encontró, en primer lugar, en la categoría de leches y derivados lácteos $(n=711) y$, en segundo lugar, en la de carnes y derivados cárnicos $(\mathrm{n}=327)$ (cuadro suplementario 1 ).

Los quesos campesinos fueron el alimento con mayor frecuencia $(31,34 \%)$ de aislamientos de L. monocytogenes. El serotipo aislado más veces en el grupo completo de alimentos, fue el $4 b(51,93 \%)$ (cuadro 1$)$.

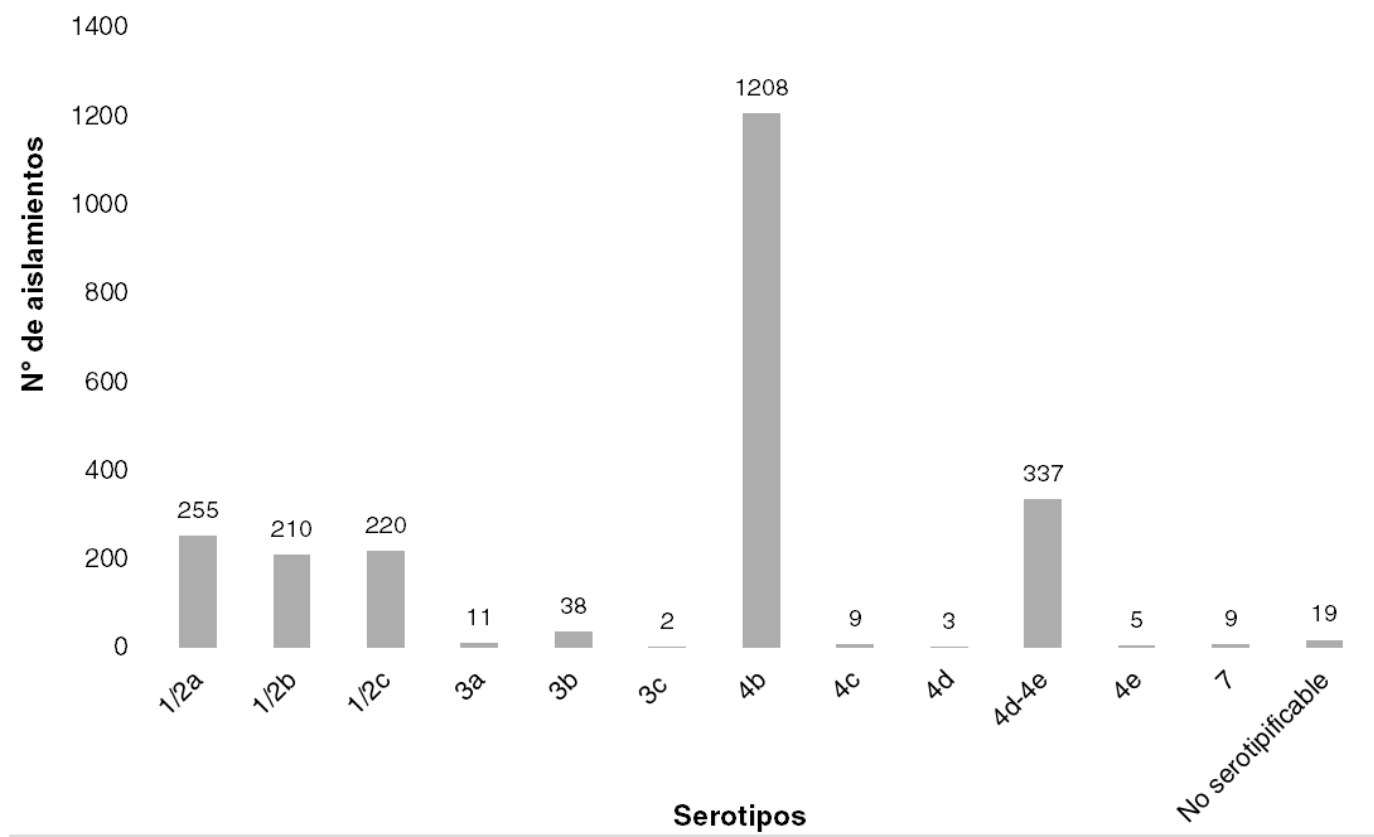

Figura 2. Determinación de serotipos de Listeria monocytogenes aislados de alimentos, Colombia, 2010-2018

Cuadro 1. Frecuencia de Listeria monocytogenes y determinación de serotipos aislados de alimentos, Colombia, 20102018

\begin{tabular}{|c|c|c|c|c|c|c|c|c|c|c|c|c|c|c|}
\hline \multirow{2}{*}{ Alimentos } & $/ 2 a$ & $/ 2 b$ & $1 / 2 c$ & $3 a$ & $3 b$ & $3 c$ & $4 b$ & $4 c$ & 4d & $4 d-4 e$ & $4 e$ & 7 & NS & Total \\
\hline & $\%$ & $\%$ & $\%$ & $\%$ & $\%$ & $\%$ & $\%$ & $\%$ & $\%$ & $\%$ & $\%$ & $\%$ & $\%$ & $\%$ \\
\hline sino & 2,24 & 2,19 & 4,56 & 0,39 & 0,77 &, 04 & 16,17 & 0,00 & 0,04 & 4,69 & 0,00 & 0,04 & 0,21 & 31,3 \\
\hline $\begin{array}{l}\text { amón co } \\
\text { ordero, }\end{array}$ & 3 & 76 & 95 & 0 & 3 & 0 & & 0 & 4 & 9 & 4 & 4 & 9 & 16 \\
\hline uaj & 0,73 & 0,26 & 1,12 & 0,00 & 0,04 & 0,00 & 2,49 & 0,00 & 0,00 & 0,60 & 0,00 & 0,00 & 4 & 5,2 \\
\hline eche & 0,52 & 0,09 & 0,04 & 0,00 & 0,04 & 0,04 & 2,71 & 0,00 & 0,00 & 0,52 & 0,00 & 0,00 & 0,00 & 3,9 \\
\hline alc & 0,39 & 0,34 & 0,21 & 0,00 & 0,17 & 0,00 & 1,16 & 0,00 & 0,04 & 0,52 & 0,00 & 0,00 & 0,00 & 2,8 \\
\hline 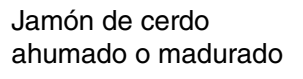 & 0 & 0,04 & 0,17 & 0 & 0,00 & 0 , & 6 & 0,00 & 0,00 & 39 & 0,00 & 0,13 & 4 & 2,84 \\
\hline ues & 0,21 & 0,26 & 0,00 & 0,00 & 0,00 & 0,00 & 1,72 & 0,04 & 0,00 & 0,30 & 0,00 & 0,04 & 0,09 & 2,6 \\
\hline $\begin{array}{l}\text { pollo, } \\
\text { o }\end{array}$ & 0,09 & 0,13 & 0,30 & 0,00 & 0,00 & 0,00 & 1,20 & 0,04 & 0,00 & 0,69 & 0,00 & 0,00 & 0,00 & 2,45 \\
\hline Morta & 0,26 & 0,09 & 0,04 & 0,00 & 0,17 & 0,00 & 1,42 & 0,00 & 0,00 & 0,39 & 0,00 & 0,04 & 0,00 & 2,4 \\
\hline insa & 0,26 & 0,17 & 0,00 & 0,00 & 0,00 & 0,00 & 1,07 & 0,00 & 0,00 & 0,34 & 0,00 & 0,00 & 0,00 & 1,8 \\
\hline Total & 6,62 & 5,33 & 7,39 & 0,39 & 1,33 & 0,09 & 35,25 & 0,17 & 0,13 & 9,72 & 0,04 & 0,30 & 0,47 & 67,24 \\
\hline Otros & 4,34 & 3,70 & 2,06 & 0,09 & 0,30 & 0,00 & 16,68 & 0,21 & 0,00 & 4,77 & 0,17 & 0,09 & 0,34 & 32,76 \\
\hline Total & 10,96 & 9,03 & 9,46 & 0,47 & 1,63 & 0,09 & 51,93 & 0,39 & 0,13 & 14,50 & 0,21 & 0,39 & 0,82 & 100,00 \\
\hline
\end{tabular}

NS: no serotipificable 
De los 368 aislamientos seleccionados para PFGE, la técnica pudo utilizarse en 333; no fue posible hacerlo en 35 (9,5\%), probablemente por la calidad del ADN. Estos aislamientos se agruparon en 167 patrones de PFGE diferentes y se designaron de LM-001 a LM-167 (figura suplementaria 1).

\section{Distribución de patrones frecuentes de PFGE}

Para el análisis de los patrones de PFGE obtenidos, se hizo una clasificación de los cuatro patrones más frecuentes, es decir, aquellos con más de 10 aislamientos (cuadro 2) (figura suplementaria 1).

Patrón LM-076. El 81,2\% (13/16) de los aislamientos pertenecía al serotipo $4 \mathrm{~b}$, el cual se presentó en siete clases diferentes de alimentos y en tres departamentos (cuadro 2) (figura suplementaria 1). Este patrón únicamente se presentó en los cinco primeros años de vigilancia.

Patrón LM-0154. El 87,5\% (14/16) de los aislamientos con este patrón correspondió al serotipo $1 / 2 \mathrm{c}$, el cual fue el más representativo, seguido por el $1 / 2 b$ y el $4 b$. El queso campesino fue el alimento en el cual se encontró con mayor frecuencia y las muestras provenían casi exclusivamente del departamento de Antioquia (14/16) (87,5\%) (cuadro 2) (figura suplementaria 1). Este patrón únicamente se presentó en los cinco primeros años de vigilancia (cuadro 3).

Cuadro 2. Distribución de perfiles de PFGE más frecuentes según tipo de alimento, Colombia, 2010-2018

\begin{tabular}{|c|c|c|c|c|c|c|c|c|c|c|}
\hline \multirow{2}{*}{ Patrón PFGE } & \multirow{2}{*}{ Alimento relacionado } & \multirow{2}{*}{$\begin{array}{c}\text { Número de } \\
\text { aislamientos por patrón }\end{array}$} & \multicolumn{7}{|c|}{ Serotipos } & \multirow{2}{*}{ Procedencia } \\
\hline & & & $1 / 2 a$ & $1 / 2 b$ & $1 / 2 c$ & $3 a$ & $3 b$ & $4 b$ & 4d NS & \\
\hline LM 076 & $\begin{array}{l}\text { Ensalada de verduras (1), } \\
\text { helado (1), jamón (3), leche } \\
\text { cruda (1), pollo crudo (1), } \\
\text { queso campesino (8) y queso } \\
\text { doble crema (1) }\end{array}$ & 16 & 1 & 0 & 0 & 0 & 0 & 13 & 0 & $\begin{array}{l}2 \text { Antioquia (9) } \\
\text { Bogotá (5) } \\
\text { Caldas (2) }\end{array}$ \\
\hline LM 154 & $\begin{array}{l}\text { Cuajada (1), jamón de cerdo } \\
\text { (1), queso campesino (14) }\end{array}$ & 16 & 0 & 1 & 14 & 0 & 0 & 1 & 00 & $\begin{array}{l}0 \text { Antioquia (14) } \\
\text { Bogotá (2) }\end{array}$ \\
\hline LM 047 & $\begin{array}{l}\text { Agua de panela (1), carne } \\
\text { cocinada (1), carne cruda } \\
\text { (1), crema de leche (1), } \\
\text { flan de leche (1), jamón (2), } \\
\text { queso campesino (2), salami } \\
\text { (1), sánduche (1), tocineta (1) }\end{array}$ & 12 & 0 & 0 & 0 & 0 & 0 & 6 & 6 & $\begin{array}{l}0 \text { Bogotá (11) } \\
\text { Valle (1) }\end{array}$ \\
\hline LM 072 & $\begin{array}{l}\text { Arroz con pollo (1), ensalada } \\
\text { de frutas (1), ensalada de } \\
\text { verduras (1), jamón cocido } \\
(1), \text { menú (1), pechuga de } \\
\text { pollo cocinada (1), queso } \\
\text { campesino (4) }\end{array}$ & 10 & 0 & 0 & 0 & 0 & 0 & 10 & 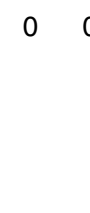 & $\begin{array}{l}0 \text { Antioquia (6) } \\
\text { Bogotá (1) } \\
\text { Caldas (1) } \\
\text { Cundinamarca (1) } \\
\text { Valle (1) }\end{array}$ \\
\hline $\begin{array}{l}\text { Otros patrones } \\
n=163\end{array}$ & $\begin{array}{l}67 \text { diferentes tipos de } \\
\text { alimentos, el queso } \\
\text { campesino concentró el } \\
\text { mayor número de muestras: } \\
69 \text { en total }\end{array}$ & 279 & 34 & 37 & 21 & 2 & 5 & 146 & 2 & $\begin{array}{l}2 \text { Antioquia (66) } \\
\text { Bogotá (108) } \\
\text { Nariño (36) } \\
\text { Valle (28) } \\
\text { Cundinamarca (9) } \\
\text { Caldas (7) } \\
\text { San Andrés (3) } \\
\text { Amazonas, Boyacá, Cauca, } \\
\text { Magdalena, Quindío, Risaralda y } \\
\text { Putumayo (2 aislamientos cada uno) } \\
\text { Arauca, Bolívar, Caquetá, Cesar, } \\
\text { Córdoba, Magdalena, Norte } \\
\text { de Santander, San Andrés (1 } \\
\text { aislamiento cada uno) }\end{array}$ \\
\hline Total de aislamientos & & 333 & 35 & 38 & 35 & 2 & 5 & 176 & 384 & 4 \\
\hline
\end{tabular}

NS: no serotipificable 
Cuadro 3. Distribución de perfiles de PFGE más frecuentes por serotipo y año en aislamientos de Listeria monocytogenes, Colombia, 2010-2018

\begin{tabular}{|c|c|c|c|c|c|c|c|c|c|c|c|c|c|c|c|c|c|c|}
\hline \multirow{3}{*}{ Año/patrón de PFGE } & \multicolumn{17}{|c|}{ Serotipos } & \multirow{3}{*}{$\begin{array}{l}\text { Número de } \\
\text { aislamientos }\end{array}$} \\
\hline & \multicolumn{2}{|c|}{$1 / 2 a$} & \multicolumn{2}{|c|}{$1 / 2 b$} & \multicolumn{2}{|c|}{$1 / 2 c$} & \multicolumn{2}{|c|}{$3 a 3 b$} & \multicolumn{4}{|c|}{$4 b$} & \multicolumn{3}{|c|}{ 4d } & \multicolumn{2}{|l|}{ NS } & \\
\hline & 76 & 0 & 154 & 0 & 154 & 0 & 0 & 0 & 47 & 727 & & 54 & 0 & 47 & 07 & 760 & 0 & \\
\hline 2010 & 0 & 5 & 1 & 11 & 2 & 1 & 1 & 2 & 0 & 0 & 1 & 1 & 32 & 3 & 7 & 00 & 0 & 67 \\
\hline 2011 & 0 & 8 & 0 & 9 & 2 & 3 & 1 & 1 & 1 & 3 & 1 & 0 & 25 & 2 & 6 & 00 & 0 & 62 \\
\hline 2012 & 0 & 5 & 0 & 3 & 4 & 5 & 0 & 2 & 1 & 0 & 5 & 0 & 20 & 0 & 2 & 11 & 1 & 49 \\
\hline 2013 & 0 & 7 & 0 & 5 & 2 & 3 & 0 & 0 & 0 & 5 & 6 & 0 & 18 & 0 & 8 & 00 & 0 & 54 \\
\hline 2014 & 1 & 4 & 0 & 6 & 4 & 6 & 0 & 0 & 0 & 1 & 0 & 0 & 23 & 1 & 8 & 11 & 1 & 56 \\
\hline 2015 & 0 & 2 & 0 & 1 & 0 & 1 & 0 & 0 & 1 & 1 & 0 & 0 & 11 & 0 & 1 & 00 & 0 & 18 \\
\hline 2016 & 0 & 0 & 0 & 1 & 0 & 1 & 0 & 0 & 0 & 0 & 0 & 0 & 7 & 0 & 0 & 00 & 0 & 9 \\
\hline 2017 & 0 & 2 & 0 & 0 & 0 & 1 & 0 & 0 & 2 & 0 & 0 & 0 & 7 & 0 & 0 & 00 & 0 & 12 \\
\hline 2018 & 0 & 1 & 0 & 1 & 0 & 0 & 0 & 0 & 1 & 0 & 0 & 0 & 3 & 0 & 0 & 00 & 0 & 6 \\
\hline Total & 1 & 34 & & 37 & 14 & 21 & 2 & 5 & 6 & 101 & 3 & 11 & 146 & 63 & 32 & 22 & 2 & 333 \\
\hline
\end{tabular}

NS: no serotipificable; patrón de PFGE: (047/072/076/154); O: otros, corresponde a 163 perfiles que contenían menos de 10 aislamientos cada uno

Patrón LM-047. Se encontraron 12 aislamientos con este patrón, seis del serotipo $4 \mathrm{~b}$ y seis del $4 \mathrm{~d}$, en 10 alimentos diferentes y casi exclusivamente $(91,6 \%)$ en Bogotá (cuadro 2) (figura suplementaria 1).

Patrón LM-072. Todos los aislamientos de este patrón fueron del serotipo $4 b$, es decir que, en las muestras evaluadas en el estudio, este patrón se detectó exclusivamente en este serotipo. Se presentó en siete clases de alimentos diferentes y en cinco departamentos, en los años 2011, 2013, 2014 y 2015 (cuadro 2) (figura suplementaria 1).

Otros patrones. Se encontraron 163 patrones diferentes de PFGE en 279 aislamientos distribuidos en 67 diferentes tipos de alimentos, de los cuales el más frecuente fue el queso campesino. Los serotipos más frecuentes en esta categoría fueron el 4b, con $146(52,4 \%)$ aislamientos, el 1/2b, con $37(13,2$ $\%)$, el 1/2a, con 34 (12,2\%), y el 4d, con $32(11,5 \%)$. Los aislamientos de esta categoría procedían de 22 departamentos (cuadro 2) (figura suplementaria 1).

\section{Distribución de patrones de PFGE por serotipo}

Serotipo 4b. De los 333 aislamientos evaluados por PFGE, más de la mitad ( $n=176 ; 52,9 \%$ ) correspondió al serotipo 4b, recuperados durante todos los años de vigilancia (cuadro 3).

Serotipo $4 d$. Se procesaron $38(11,4 \%)$ aislamientos recuperados en el 2010, el 2011 y el 2014 (cuadro 3).

Serotipo 1/2b. Se procesaron 38 (11,4 \%) aislamientos recuperados en todos los años, excepto en el 2017. Solo uno se relacionó con los patrones frecuentes.

Serotipos 1/2a y 1/2c. Del serotipo 1/2a se analizaron 35 (10,5\%) aislamientos encontrados en todos los años de estudio, excepto en el 2016, en tanto que el serotipo $1 / 2 \mathrm{c}$ se evaluó en todos los años de estudio, excepto en el 2018 (cuadro 3).

Otros serotipos. Los 11 aislamientos restantes correspondieron a los serotipos $3 b(1,5 \%)$ y $3 a(0,6 \%)$, y cuatro $(1,2 \%)$ no pudieron ser serotipificados (cuadro 3 ). Del serotipo $4 \mathrm{c}$ no se evaluaron aislamientos con la PFGE. 


\section{Patrones de PFGE en aislamientos causantes de enfermedades transmitidas por alimentos}

Los 38 aislamientos clasificados en esta categoría fueron relacionados epidemiológicamente con enfermedades transmitidas por alimentos por los laboratorios de salud pública. Se evaluaron 35 aislamientos mediante PFGE, los cuales se distribuyeron en cuatro serotipos y 30 patrones.

Serotipo 1/2a. Se encontraron cuatro aislamientos distribuidos en cuatro patrones de PFGE.

Serotipo 1/2b. Se encontraron cuatro aislamientos distribuidos en cuatro patrones de PFGE.

Serotipo 1/2c. Se encontraron siete aislamientos distribuidos en cinco patrones de PFGE.

Serotipo $4 b$. Se encontraron 20 aislamientos distribuidos en 18 patrones de PFGE (figura 3). Los serotipos $3 a, 3 b, 4 c$ y $4 d$ no se relacionaron con enfermedades transmitidas por alimentos.

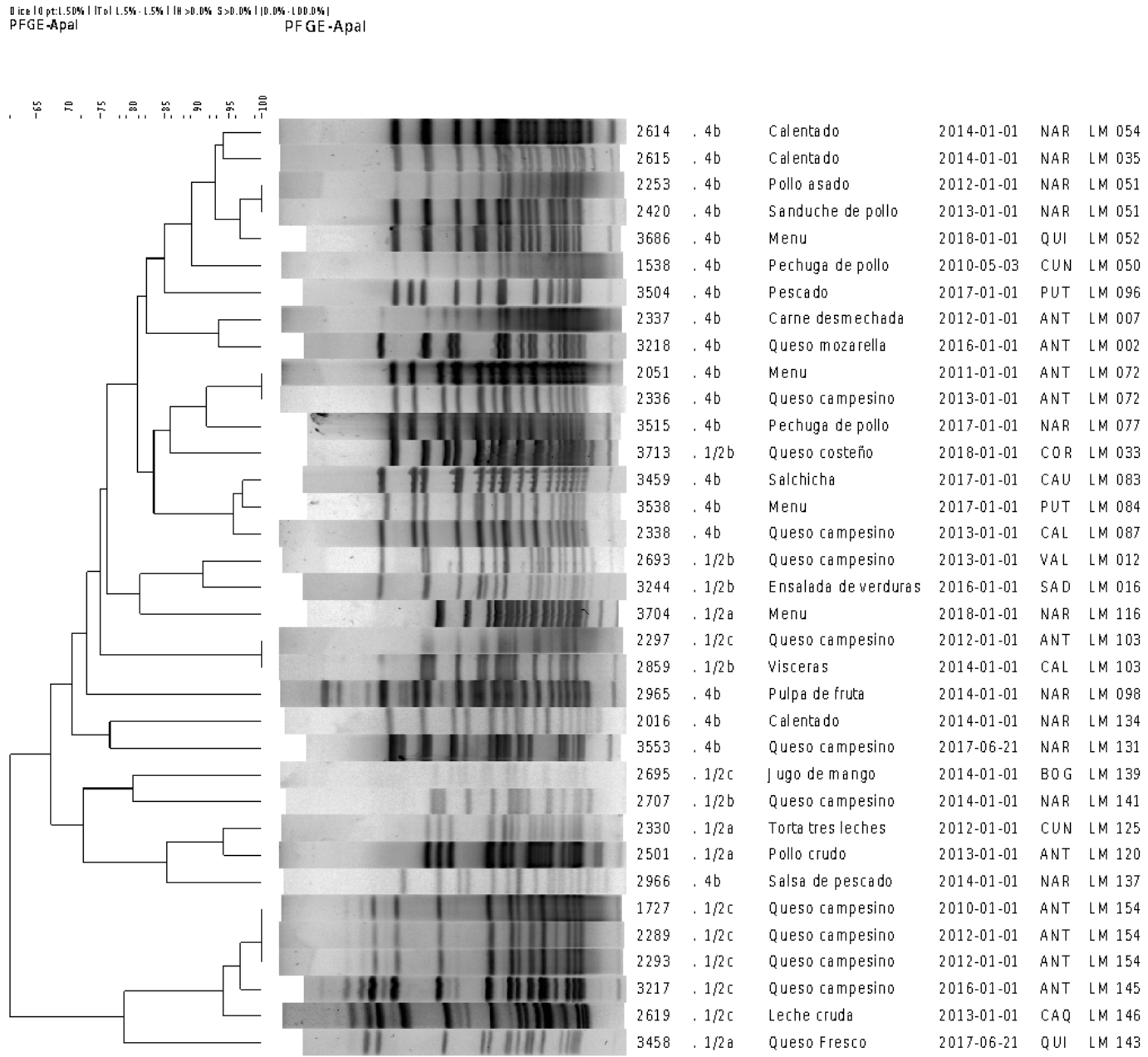

Figura 3. Patrones de PFGE de aislamientos de Listeria monocytogenes relacionados con brotes de enfermedades transmitidas por alimentos, Colombia, 2010-2018 
Los brotes de enfermedades transmitidas por alimentos se relacionaron con las categorías de alimentos preparados (arroz con pollo, calentado, carne desmechada, jugo, tortas), derivados lácteos (leche cruda, queso) y derivados cárnicos (pollo) (figura 3). Los departamentos que enviaron mayor cantidad de aislamientos relacionados con este tipo de enfermedades durante este periodo de estudio, fueron Antioquia y Nariño.

Dos aislamientos del serotipo 4b presentaron el patrón LM-51 y ambos provenían del departamento de Nariño; se encontraron en alimentos que contenían pollo y se enviaron en dos años diferentes, lo cual demuestra la persistencia en el tiempo de este patrón y su relación con enfermedades transmitidas por alimentos.

Una situación similar se presentó en Antioquia con el patrón 072, el cual se recuperó en el 2011 y en el 2013 en alimentos relacionados con estas enfermedades; el patrón LM-154 se encontró en tres aislamientos con el serotipo $1 / 2 c$, todos procedentes de queso campesino en dos años diferentes.

\section{Discusión}

En Colombia, la autoridad sanitaria ha incluido L. monocytogenes en la vigilancia rutinaria de los alimentos como indicador de inocuidad, por ser un microorganismo causante de enfermedades transmitidas por alimentos, aunque aún no se encuentra regulado en las normas nacionales.

Los serotipos encontrados en este estudio, el $4 b$, el 1/2a y el 1/2b, coinciden con los reportados a nivel mundial relacionados con casos o brotes de listeriosis $(1,21,23,24,26,28)$. Según el ciclo evolutivo de $L$. monocytogenes, hay cuatro linajes. Los serotipos $4 \mathrm{~b}$ y $1 / 2 \mathrm{~b}$ pertenecen al linaje I y son causantes de la mayoría de las listeriosis humanas transmitidas por alimentos; el serotipo1/2a pertenece al linaje II, implicado en brotes de listeriosis humanas y también aislado del ambiente y de alimentos (49). Aunque no todos los serotipos de L. monocytogenes tienen factores de virulencia similares, para efectos de la salud pública, todos ellos se consideran potencialmente patógenos $(1,23,26)$.

En la vigilancia rutinaria de alimentos, Bogotá fue la entidad territorial que envió el mayor número de aislamientos de L. monocytogenes, seguida por Antioquia, lo que posiblemente se debe a que existe un mejor programa de vigilancia de este agente patógeno en estas dos seccionales.

Los derivados lácteos, principalmente el queso campesino y las cuajadas, fue el grupo de alimentos en el que más se aisló L. monocytogenes, principalmente del serotipo $4 \mathrm{~b}$, lo cual también puede deberse a que muchos son de producción artesanal, en la cual es más común el uso de leches no pasteurizadas, así como la ausencia de buenas prácticas de manufactura.

En los derivados cárnicos, las concentraciones de nitritos permitidas como conservantes no inhiben a L. monocytogenes (31) y permiten su crecimiento. En el presente estudio se reportaron aislamientos, principalmente del serotipo $4 b$, en jamones cocidos de cerdo, cordero, pavo y pollo. Es probable que esto se deba a fallas en el mantenimiento de la higiene a lo largo de la cadena de producción, conservación, transporte, fraccionamiento y manipulación en la venta de estos productos, lo cual, unido a características como el gran contenido de nutrientes, la importante actividad del agua (AW) $(\geq 0,92 \%)$, un $\mathrm{pH}$ de 4,4 o menos, y la conservación prolongada a bajas temperaturas de refrigeración, permite la supervivencia de L. monocytogenes (50). 
En comparación con un reporte previo de la distribución de serotipos de $L$. monocytogenes en alimentos en Colombia (51), se encontró que no se han presentado variaciones en el tipo de alimento contaminado con este agente patógeno con respecto a los años anteriores y, tampoco, en los serotipos encontrados, lo cual pone en evidencia la falta de intervención en los establecimientos que son de obligatoria vigilancia y control.

Se ha sugerido que en los aislamientos de alimentos se encuentran principalmente los serotipos $1 / 2 a, 1 / 2 b$ y $1 / 2 c(1,21)$. En el presente estudio, se observó con mayor frecuencia el serotipo $4 \mathrm{~b}$, de gran virulencia y causante de la enfermedad en humanos $(1,21,23,26)$, como ya lo reportaron Montero, et al. (52), quienes evaluaron los serotipos de L. monocytogenes aislados de alimentos listos para el consumo y encontraron una asociación del serotipo $4 \mathrm{~b}$ con alimentos como el queso y los mariscos congelados. Esto puede deberse a que pertenecen a uno de los clones epidémicos identificados que circulan en el mundo, característica que debería evaluarse en estudios posteriores; además, cobra importancia porque uno de los patrones frecuentes encontrados en este estudio, el LM 072, se asoció exclusivamente con aislamientos de serotipo $4 \mathrm{~b}$ provenientes de alimentos listos para el consumo, como arroz con pollo, ensalada de frutas y verduras, menú listo para consumir, pechuga de pollo cocinada y queso campesino. Ello evidencia la necesidad de incluir a la ausencia de L. monocytogenes como parámetro de inocuidad obligatorio, principalmente en la categoría de alimentos listos para el consumo.

Se ha reportado que los aislamientos de origen alimentario tienen mayor diversidad genética que los de origen humano (53). Esta característica también se observó en el presente estudio, en el cual se obtuvieron más de 160 perfiles diferentes de electroforesis, lo que sugiere diversas fuentes y condiciones de selección para los genotipos específicos encontrados en alimentos y ambientes de plantas de producción; además, sugiere que solo ciertos grupos de alimentos están relacionados con la enfermedad en humanos $(54,55)$.

Los aislamientos caracterizados mediante PFGE permitieron identificar patrones moleculares que pueden indicar contaminación y persistencia de clones en el tiempo. La persistencia de ciertos genotipos en las instalaciones destinadas a la producción de alimentos, puede ser un signo de fallas en el control del proceso de fabricación o indicar resistencia de los microorganismos a los métodos de limpieza utilizados. Tal persistencia puede deberse a la supervivencia y crecimiento de ciertas cepas en biopelículas o nichos dentro del entorno alimentario, las cuales pueden ser difíciles de limpiar y desinfectar, así como a la reintroducción repetida de tales cepas del ambiente externo en las instalaciones de procesamiento de alimentos a lo largo del tiempo (54).

La cantidad de patrones caracterizados como no persistentes, que en este trabajo se clasificaron como "otros", indica la diversidad de aislamientos que existen en el entorno del procesamiento de alimentos, así como una probable contaminación esporádica continua, lo que indicaría falta de cumplimiento de los protocolos de higiene y limpieza y, por lo tanto, el riesgo de contaminación de los productos alimenticios (54).

Los patrones de PFGE clasificados como más frecuentes en este estudio, se presentaron por lo menos una vez en la mayoría de los años en varios departamentos y en alimentos listos para consumir, como quesos, carnes, comidas preparadas y sánduches, entre otros. La permanencia de estos patrones de PFGE en el tiempo, sugiere que los aislamientos de $L$. 
monocytogenes implicados tienen un reservorio permanente en los intervalos de tiempo, por lo general largos, entre sus apariciones $(53,54)$.

Este estudio brinda información importante sobre la epidemiología de $L$. monocytogenes y puede ayudar a adoptar decisiones sanitarias en Colombia, así como servir de base para estudios posteriores de identificación de los clones epidémicos. Consideramos que los resultados de la genotipificación se pueden utilizar como información de base para hacer análisis genéticos ampliados, por ejemplo, la secuenciación completa del genoma de $L$. monocytogenes.

La clasificación de algunos alimentos, como los 'menús', se dificultó porque la información enviada al laboratorio no estaba completa. Los resultados obtenidos indican la necesidad de implementar buenas prácticas de manufactura con procedimientos estandarizados, en las fábricas artesanales del país (20); además, que las autoridades sanitarias del país deben continuar implementando programas eficaces de vigilancia frente a la prohibición de la venta informal de leche cruda, y de la de quesos o cuajadas elaboradas con leche sin pasteurizar.

Se recomienda, al Ministerio de Salud y Protección Social, incluir la listeriosis entre las enfermedades de notificación obligatoria. Asimismo, incluir en la legislación colombiana la ausencia de L. monocytogenes como parámetro de inocuidad en el análisis de los alimentos que, por sus características intrínsecas y extrínsecas, permiten su crecimiento, como es el caso de aquellos listos para el consumo.

\section{Agradecimientos}

A los laboratorios de salud pública de Arauca, Antioquia, Atlántico, Bolívar, Boyacá, Caldas, Caquetá, Cauca, Casanare, Cesar, Córdoba, Cundinamarca, Bogotá, La Guajira, Magdalena, Meta, Nariño, Norte de Santander, Putumayo, Quindío, Risaralda, Santander, Sucre, San Andrés Islas y Valle del Cauca; y al laboratorio de referencia para Listeria del Invima, por su activa participación en la Red Nacional de Vigilancia Microbiológica de Listeria y por la recuperación de los aislamientos de L. monocytogenes que hicieron posible este estudio. A Marc Lecuit y Alexandre Leclercq, del Centro Nacional de Referencia y del Centro Colaborativo de la OMS para Listeria, Instituto Pasteur (París, Francia), por su contribución en el desarrollo de este estudio mediante la implementación del control externo de calidad de los genogrupos de L. monocytogenes. A Jocelyne Rocourt, exjefe del Laboratorio de Listeria del Instituto Pasteur de París y del Centro Colaborativo de la OMS para la listeriosis de origen alimentario, por la donación de cepas de la colección de Listeria del Instituto Pasteur utilizadas en la producción de los sueros de listeria destinados a la serotipificación y la PFGE de L. monocytogenes en nuestro laboratorio. A Óscar Eduardo Pacheco García, subdirector de Prevención, Vigilancia y Control en Salud Pública del Instituto Nacional de Salud, por la corrección y la lectura crítica del presente artículo.

\section{Referencias}

1. Vásquez-Boland JA, Kuhn M, Berche P, Chakraborty T, Domínguez-Bernal G, Goebel W, et al. Listeria pathogenesis and molecular virulence determinants. Clin Microbiol Rev. 2001;14:584-640. https://doi:10.1128/CMR.14.3.584-640.2001

2. Murray EGD, Webb RA, Swann MBR. A disease of rabbits characterized by large mononuclear leukocytosis cause by a hitherto undescribed bacillus, Bacterium monocytogenes. J Path Bact. 1926;29:407-34. https://doi.org/10.1002/path.1700290409 
3. Seeliger HP. Nonpathogenic Listeriae: L. innocua sp.n. Zentralbl Bakteriol Mikrobiol Hyg A. 1981;24:487-93. https://loi.org/10.1016/S0174-3031(81)80108-4

4. Rocourt J, Grimont PAD. Listeria welshimeri sp. nov. and Listeria seeligeri sp. nov. Int J Syst Evol Microbiol.1983;33:866-9. https://doi.org/10.1099/00207713-33-4-866

5. Rocourt J, Boerlin P, Grimont F, Jacquet C, Piffaretti JC. Assignment of Listeria grayi and Listeria murrayi to a single species, Listeria grayi, with a revised description of Listeria grayi. Int J Syst Evol Microbiol.1992;42:171-4. https://doi.org/10.1099/00207713-42-1-171

6. Seeliger HPR, Rocourt J, Schrettenbrunner A, Grimont PAD, Jones D. Listeria ivanovii sp. nov. Int J Syst Evol Microbiol.1984;34:336-7. https://doi.org/10.1099/00207713-34-3-336

7. Leclercq A, Clermont $\mathrm{D}$, Bizet $\mathrm{CH}$, Grimont $\mathrm{P}$, Le-Fléche-Matéos $\mathrm{A}$, Roche $\mathrm{S}$, et al. Listeria rocourtiae sp. nov. Int J Syst Evol Microbiol. 2010;60:2210-4. https://doi.org/10.1099/ijs.0.017376-0

8. Graves LM, Helsel LO, Steigerwalt AG, Morey RE, Daneshvar MI, Roof SE, et al. Listeria marthii sp. nov. isolated from the natural environment, Finger Lakes National Forest. Int $\mathrm{J}$ Syst Evol Microbiol. 2010;60:1280-8. https://doi.org/10.1099/ijs.0.014118-0

9. Den Bakker HC, Warchocki S, Wrigt EM, Allred AF, Ahlstrom C, Manuel CS, et al. Listeria floridensis sp. nov., Listeria aquatic sp. nov., Listeria cornellensis sp. nov., Listeria riparia sp. nov. and Listeria grandensis sp. nov., from agricultural and natural environments. Int J Syst Evol Microbiol. 2014;64:1882-9. https://doi.org/10.1099/ijs.0.052720-0

10. Weller D, Andrus A, Wiedmann M, Den Bakker HC. Listeria booriae sp. nov. and Listeria newyorkensis sp. nov., from food processing environments in the USA. Int J Syst Evol Microbiol. 2015;65:286-92. https://doi.org/10.1099/ijs.0.070839-0

11. Bertsch D, Rau J, Eugster MR, Haug MC, Lawson PA, Lacroix C, et al. Listeria fleischmannii sp. nov., isolated from cheese. Int J Syst Evol Microbiol. 2013;63:526-32. https://doi.org/10.1099/ijs.0.036947-0

12. Halter EL, Neuhaus K, Scherer S. Listeria weihenstephanensis sp. nov., isolated from the water plant Lemna trisulca taken from a freshwater pond. Int J Syst Evol Microbiol. 2013;63:641-7. https://doi.org/10.1099/ijs.0.036830-0

13. Núñez-Montero K, Leclercq A, Moura A, Vales G, Peraza J, Pizarro-Cerdá J, et al. Listeria costaricencis sp. nov. Int J Syst Evol Microbiol. 2018;68:844-50 https://doi.org/10.1099/ijsem.0.002596

14. Doijad SP, Poharkar KV, Kale SB, Kerkar S, Kalorey DR, Kurkure NV, et al. Listeria goaensis sp. nov. Int J Syst Evol Microbiol. 2018;68:3285-91. https://doi.org/10.1099/ijsem.0.002980

15. Leclercq A, Moura A, Vales G, Thessaud-Rita N, Aguilhon C, Lecuit M. Listeria thailandesis sp. nov. Int J Syst Evol Microbiol 2019;69:74-81. https://doi.org/10.1099/ijsem.0.003097

16. Quereda JJ, Leclercq A, Moura A, Vales G, Gómez-Martín A, García-Muñoz A, et al. Listeria valentina sp. nov., isolated from a water trough and the faeces of healthy sheeps. Int J Syst Evol Microbiol. 2020;70:5868-79. https://doi.org/10.1099/ijsem.0.004494

17. Carlin CR, Liao J, Weller D, Gou X, Orsi R, Wiedmann M. Listeria cossartiae sp.nov., Listeria farberi sp. nov., Listeria immoviles sp.nov., Listeria portnoyi sp. nov., and Listeria rustica sp. nov., isolated from agricultural water and natural environments. Int J Syst Evol Microbiol. 2021;71:004795. https://doi.org/10.1099/ijsem.0.004795

18. Guillet C, Join-Lambert O, Le Monnier A, Leclercq A, Mechaï F, Manzer-Bruneel MF, et al. Human listeriosis caused by Listeria ivanovii. Emerg Infect Dis. 2010;16:136-8. https://doi.org/10.3201/eid1601.091155

19. Favaro M, Sarmati L, Sancesario G, Fontana C. First case of Listeria innocua meningitis in a patient on steroids and eternecept. JMM Case Rep. 2014:1. https://doi.org/10.1099/jmmcr.0.003103

20. McIntyre L, Wilcott L, Naus M. Listeriosis outbreaks in British Columbia, Canada, caused by soft ripened cheese contaminated from environmental sources. Biomed Res Int. 2015;2015:131623. https://doi.org/10.1155/2015/131623

21. Pan Y, Breidt F Jr, Kathariou S. Competition of Listeria monocytogenes serotype 1/2a and 4b strains in mixed-culture biofilms. Appl Environ Microbiol. 2009;75:5846-52. https://doi.org/10.1128/AEM.00816-09

22. Jemmi T, Stephan R. Listeria monocytogenes: Food-borne pathogen and hygiene indicator. Rev Sci Tech. 2006;25:571-80. 
23. Velge P, Roche SM. Variability of Listeria monocytogenes virulence: A result of the evolution between saprophytism and virulence? Fut Microbiol. 2010;5:1799-821. https://doi.org/10.2217/fmb.10.134

24. Charlier C, Fevre C, Travier L, Cazenave B, Bracq-Dieye H, Podevin J, et al. Listeria monocytogenes-associated biliary tract infections: A study of 12 consecutive cases and review. Medicine (Baltimore). 2014;93:e105. https://doi.org/10.1097/MD.0000000000000105

25. Centers for Disease Control and Prevention (CDC). Listeria (Listeriosis). Symptoms. Fecha de consulta: 24 de julio de 2019. Disponible en: https://www.cdc.gov/listeria/index.html

26. Doumith M, Cazalet C, Simoes N, Frangeul L, Jacquet C, Kunts F, et al. New aspects regarding evolution and virulence of Listeria monocytogenes revealed by comparative genomics and DNA arrays. Infect Immun. 2004;72:1072-83. https://doi.org/10.1128/IAl.72.2.1072-1083.2004

27. Vélez-Leal JL, Dávila-Ramírez F. Listeriosis neonatal en Colombia... ¿Igual que hace veinte años? Rev Cien Salud. 2015;13:301-8. https://doi.org/10.12804/revsalud13.02.2015.11

28. Laksanalamai P, Steyert SR, Burall LS, Datta AR. Genome sequences of Listeria monocytogenes serotype $4 \mathrm{~b}$ variant strains isolated from clinical and environmental sources. Genome Announc. 2013;1:e00771-13. https://doi.org/10.1128/genomeA.00771-13

29. Linnan MJ, Mascola L, Lou XD, Goulet V, May S, Salminen C, et al. Epidemic listeriosis associated with Mexican-style cheese. N Engl J Med. 1988;319:823-8. https://doi.org/10.1056/NEJM198809293191303

30. Bille J, Blanc DS, Schmid H, Boubaker K, Baumgartner A, Siegrist HH, et al. Outbreaks of human listeriosis associated with tomme cheese in northwest Switzerland 2005. Euro Surveill. 2006;11:91-3. https://doi.org/10.2807/esm.11.06.00633-en

31. Rossi ML, Paiva A, Tornese M, Chianelli S, Troncoso A. Listeria monocytogenes outbreaks: A review of the routes that favour bacterial presence. Rev Chil Infect. 2008;25:328-35. https://doi.org/10.4067/S0716-10182008000500002

32. Centers for Disease Control and Prevention (CDC). Outbreak of Listeria monocytogenes infections associated with pasteurized milk from a local dairy -Massachusetts, 2007. Morb Mortal Wkly Rep. 2008;57:1097-100.

33. Lomonaco S, Verghese B, Gerner-Smith P, Tarr C, Gladney L, Lavin J, et al. Novel epidemic clones of Listeria monocytogenes, United States, 2011. Emerg Infect Dis. 2013;19:147-50. https://doi.org/10.3201/eid1901.121167

34. Graves LM, Hunter SB, Ong AR, Schoonmaker-Bopp D, Hise K, Kornstein L, et al. Microbiological aspects of the investigation that traced the 1998 outbreak of listeriosis in the United States to contaminated hot dogs and establishment of molecular subtypingbased surveillance for Listeria monocytogenes in the Pulse Net Network. J Clin Microbiol. 2005;43:2350-5. https://doi.org/10.1128/JCM.43.5.2350-2355.2005

35. Brett MSY, Short P, McLauchlin J. A small outbreak of listeriosis associated with smoked mussels. Int J Food Microbiol.1998;43:223-9. https://doi.org/10.1016/S0168-1605(98)00116-0

36. Desai AN, Anyoba A, Madoff LC, Lasmmann B. Changing epidemiology of Listeria monocytogenes outbreaks, sporadic cases, and recalls globally: A review of ProMED reports from1.996 to 2018. Inter J Infect Dis. 2019;84:48-53. https://doi.org/10.1016/j.ijid.2019.04.021

37. Food Drug Administration (FDA). Bacteriologycal analytical manual online. Detection and enumeration of Listeria monocytogenes in foods. Washington, D.C.: FDA; 2017. p.1-19. Fecha de consulta: 24 de julio de 2018. Disponible en: https://www.fda.gov/food/laboratorymethods-food/bacteriological-analytical-manual-bam

38. Seeliger HPR, Höhne K. Serotyping of Listeria monocytogenes and related species. En: Bergan T, Norris JR, editors. Methods in Microbiology. 1979;13:31-49. https://doi.org/10.1016/S0580-9517(08)70372-6

39. Seeliger HP, Langer B. Serological analysis of the genus Listeria. Its values and limitations. Inter J Food Microbiol. 1989;8:245-8. https://doi.org/10.1016/0168-1605(89)90020-2

40. Bille J, Rocourt J. WHO international multicenter Listeria monocytogenes subtyping studyrationale and set-up of the study. Int J Food Microbiol. 1996;32:251-62. https://doi.org/10.1016/S0168-1605(96)01140-3

41. Instituto Nacional de Salud. Dirección de Vigilancia y Análisis del Riesgo en Salud Pública. Grupo Gestión del Riesgo. Base de datos, 2010-2018. Bogotá: SIVIGILA; Instituto Nacional de Salud, 2019. 
42. Ministerio de Salud. Instituto Nacional de Salud. SIVIGILA-SIANIESP. Sistema de información de apoyo a la notificación e investigación de eventos de interés en salud pública. Registros individuales de prestación de servicios en Colombia años 2010-2018. Bogotá: Instituto Nacional de Salud, 2018.

43. Instituto Nacional de Salud. Dirección Vigilancia y Análisis del Riesgo en Salud Pública, Subdirección de Prevención, Vigilancia y Control en Salud Pública. Protocolo de Vigilancia en salud pública: meningitis bacteriana. Bogotá: Instituto Nacional de Salud, 2014.

44. Institut Pasteur. Centre National de Référence des Listeria. Centre collaborateur de L'OMS pour la listeriose d'origene alimentaire. Laboratoire des Listeria. Protocole de serotypie. Paris: Institut Pasteur; 1997. p. 22.

45. Centers for Diseases Control and Prevention (CDC). Standard operating procedure for PulseNet PFGE of Listeria monocytogenes. 2017. Fecha de consulta: 24 de septiembre de 2019. Disponible en: https://www.cdc.gov/pulsenet/pdf/listeria-pfge-protocol-508c.pdf

46. Fugett EB, Schoonmaker-Bopp D, Dumas NB, Corby J, Wiedmann M. Pulsed-field gel electrophoresis (PFGE) analysis of temporally matched Listeria monocytogenes isolates from human clinical cases, foods, ruminant farms, and urban and natural environments reveals source-associated as well as widely distributed PFGE types. J Clin Microbiol. 2007;45:865-73. https://doi.org/10.1128/JCM.01285-06

47. Londero A, Costa M, Sucari A, Leotta G. Comparison of three molecular subtyping techniques for Listeria monocytogenes. Rev Argent Microbiol. 2019;51:359-62. https://doi.org/10.1016/j.ram.2019.01.003

48. Grif K, Heller I, Wagner M, Dierich M, Würzner R. A comparison of Listeria monocytogenes serovar $4 \mathrm{~b}$ isolates of clinical and food origin in Austria by automated ribotyping and pulsedfield gel electrophoresis. Foodborne Pathog Dis. 2006;3:138-41. https://doi.org/10.1089/fpd.2006.3.138

49. Orsi RH, Den Bakker HC, Wiedmann M. Listeria monocytogenes lineages: Genomics, evolution, ecology, and phenotypic characteristics. Int Med Microbiol. 2011;301:79-96. https://dx.doi.org/10.1016/j.ijmm.2010.05.002

50. Food and Drug Administration (FDA). Evaluation and definition of potentially hazardous foods. Factors that influence microbial growth. Washington: IFT/FDA; 2003. p. 21-32.

51. Muñoz Al. Distribution of Listeria monocytogenes serotypes isolated from foods, Colombia, 2000-2009. Biomédica. 2012;32:408-17. https://doi.org/10.7705/biomedica.v32i3.709

52. Montero D, Bodero M, Riveros G, Lapierre L, Gaggars A, Vidal M, et al. Molecular epidemiology and genetic diversity of Listeria monocytogenes isolates from a wide variety of ready-to-eat foods and their relationship to clinical strains from listeriosis outbreaks in Chile. Front Microbiol. 20015;6:384. https://doi.org/10.3389/fmicb.2015.00384

53. Bibb WF, Gellin BG, Weaver R, Schwartz B, Plikaytis BD, Reeves MW, et al. Analysis of clinical and food-borne isolates of Listeria monocytogenes in the United States by multilocus enzyme electrophoresis and application of the method to epidemiologic investigations. Appl Environ Microbiol.1990;56:2133-41. https://doi.org/10.1128/AEM.56.7.2133-2141.1990

54. Kathariou S. Listeria monocytogenes virulence and pathogenicity, a food safety perspective. J Food Prot. 2002;65:1811-29. https://doi.org/10.4315/0362-028x-65.11.1811

55. Buchanan RL, Gorris LGM, Hayman MM, Jackson TC, Whiting RC. A review of Listeria monocytogenes: An update on outbreaks, virulence, dose-response, ecology, and risk assessments. Food Control. 2017:75:1-13. https://doi.org/10.1016/j.foodcont.2016.12.016 